\title{
G s.mastaw \\ Dysfunction of Decidual Macrophages Is Apotential Risk Factor in the Occurrence of Preeclampsia
}

\section{Miaomiao Rong}

Shandong Provincial Key Laboratory of Animal Resistance Biology, College of Life Sciences, Shandong Normal University, Ji'nan, Shandong, China

\section{Xingyu Yan}

School of Medicine, Xiamen University, Xiamen, 361102, Fujian, China

\section{Hongya Zhang}

Shandong Provincial Key Laboratory of Animal Resistance Biology, College of Life Sciences, Shandong Normal University, Ji'nan, Shandong, China

\section{Chan Zhou}

Shandong Provincial Key Laboratory of Animal Resistance Biology, College of Life Sciences, Shandong Normal University, Ji'nan, Shandong, China

\section{Cong Zhang ( $\nabla$ zhangxinyunlife@163.com )}

Shandong Provincial Key Laboratory of Animal Resistance Biology, College of Life Sciences, Shandong Normal University, Ji'nan, Shandong, China. Center for Reproductive Medicine, Ren Ji Hospital, School of Medicine, Shanghai Jiao Tong University, Shanghai, https://orcid.org/0000-0002-9650-4398

\section{Research}

Keywords: preeclampsia, decidual macrophages, immune

Posted Date: January 7th, 2021

DOl: https://doi.org/10.21203/rs.3.rs-139869/v1

License: (c) (i) This work is licensed under a Creative Commons Attribution 4.0 International License. Read Full License 


\section{Abstract}

Background: Preeclampsia is a multi-factorial and multi-genetic disorder that affects more than eight million mother and baby pairs each year. Currently, most of the attention to the pathogenesis of preeclampsia has been focused on placenta, but recent progresses suggest that excellent decidualization lays foundation for placentation and growth. Moreover, preeclampsia is associated with an imbalance in immunoregulatory mechanisms, however, how the immune regulatory system in the decidua affects preeclampsia is still unclear.

Methods: In our study, we used conventional expression profiling to compare the expression and function of differential genes contained in decidua parietalis of the preeclampsia and normal human. After intersecting the genes differentially expressed between preeclampsia and the control and the genes contained in the ligand receptor network, we further explored their involvement in cell-to-cell interactions using CellPhoneDB analysis. We finally used single-cell sequencing to detect interactions between decidual cells.

Results: After intersecting the genes of differentially expressed between preeclampsia and the control gotten by conventional sequencing and the genes contained in the ligand receptor network, we found eight differentially expressed genes in a ligand-receptor relationship, and the eight genes have a characteristic: most of them participate in the interaction between decidual macrophages and other decidual immune cells. The results of single-cell sequencing of decidual cells further demonstrated that decidual macrophages affect the functions of other immune cells through export. As a result, abnormal gene expression affects the export function of decidual macrophages, which in turn affects the interaction of decidual macrophages with other immune cells.

Conclusions: The abnormal export function of decidual macrophages affects the function of immune cells interacting with them, thereby destroying the original immune regulation mechanism, and ultimately leading to the occurrence of preeclampsia.

\section{Introduction}

Preeclampsia (PE)is a pregnancy-related syndrome with high blood pressure $(\geq 140 / 90 \mathrm{mmHg}$ ) and/or proteinuria ( $\geq 0.3 \mathrm{~g} / \mathrm{L}$ ) [1]. It is a human-specific disease that can severely cause uteroplacental dysfunction, intrauterine growth restriction, and premature birth, and is one of the major causes of maternal and fetal morbidity and mortality [2]. So far, the only and most reliable treatment for PE is delivery, but the pathogenesis of PE is still an enigma. About the pathogenesis, two-stage theory is now

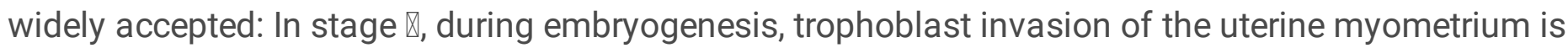
inadequate and the uterine spiral artery remodeling is impaired, which leads to placental ischemia and hypoxia metabolism disorder; In stage $\rrbracket$, the presence of a large number of undesirable factors in the maternal blood leads to a systemic inflammatory response and vascular endothelial dysfunction in the maternal body, which eventually results in PE [3-5]. 
Recent study in the pathogenesis of PE demonstrated that decidualization is closely related to the occurrence of PE. Decidualization is a transformation that endometrial stromal cells must undergo in order to adapt to pregnancy, by affecting trophoblast invasion, embryo implantation [6]. It firstly begins near spiral artery and then gradually spreads to all endometrium [7]. Decidualization is an extremely complex process in which many cytokines and hormones cooperate to promote endometrial stromal cells differentiating into decidual cells [8]. After decidualization, endometrium transforms into decidua and secretes insulin-like growth factor binding protein 1 (IGFBP1) and prolactin (PRL), both of which are thus recognized as the marker molecules of decidualization, along with the changes such as the thickening of the endometrium and the enlargement and rounding of the endometrial stromal cells $[9,10]$. Furthermore, the decidua contains a large number of pregnancy-related immunomodulatory cells that work together to form immune tolerance to pregnancy, which makes the decidua becomes an important site where the maternal immune system develops tolerance to fetal antigens. Aberrant frequency and function of decidual immune cells have been reported in a variety of obstetric complications, including PE, recurrent pregnancy loss, and preterm delivery $[11,12]$.

From an immunological point of view, the fetus is a homograft to the mother. Despite the antigenic differences between the mother and fetus, the maternal immune system does not reject it and protects it to develop normally, indicating that the immune system plays an important role in the normal course of pregnancy $[13,14]$. Decidual immune cells are important "enforcers" of maternal-fetal immune regulation, including decidual natural killer cells (dNK), decidual macrophages (dM), T cells, and to a lesser extent, dendritic cells (DC) and B cells. These immune cells converge locally in the uterus, forming a unique mother-fetus immune microenvironment [15-18].

As a subpopulation of mononuclear phagocytes of innate immune system, $\mathrm{dM}$ is highly plastic and heterogeneous. It can be classified into M1/M2 cells based on induction factors, phenotype and function [19]. $M 1$ cells have high expression of $C D 80, C D 86$, and $M H C \otimes$, produce pro-inflammatory factors, and have a strong antigen-presenting ability, thus promoting Th1-type immune responses. M2 cells highly express CD206, CD163, CD209, etc., which produce anti-inflammatory factors and have strong immunosuppressive ability so as to promote Th2-type immune response to exert immunomodulatory function [20]. Abnormalities in the phenotype and function of dM would cause an imbalance in the microenvironment at the mother-fetus interface and disruption of immune tolerance, which in turn would lead to dysfunctional trophoblast invasion and defective spiral artery reconstitution, ultimately result in $P E$ [21]. But the detailed mechanism by which dM causes $P E$ remains unclear.

In this study, we used the newly obtained single-cell sequencing data to investigate the pathogenesis of $\mathrm{PE}$, and found that the export role of $\mathrm{dM}$ was crucial, since most of the differentially expressed genes in the decidua parietalis of PE gotten by intersecting the genes found though expression profile analysis and the genes contained in the ligand receptor network are in the export function of $\mathrm{dM}$, so that the aberrant function of $\mathrm{dM}$ is an important cause in the development of $\mathrm{PE}$.

\section{Materials And Methods}




\subsection{Data collection}

We downloaded the raw data of single-cell sequencing and the gene expression matrix from Gene Expression Omnibus (GEO, https://www.ncbi.nlm.nih.gov/geo/). The original data source for single cells is GSE130560. The relevant expression profile of the decidua parietalis is derived from GSE94643 in the GEO database.

\subsection{Data processing, quality control and standardization}

We firstly used the Seurat package in the R software to process the original data count array [22]. Then we used the "NormalizeData" function to normalize the gene expression data. Afterwards, the UMI and mitochondrial gene expression values detected by the "ScaleData" function were used to correct the interbatch difference by regression analysis, and the corrected expression matrix was then used for cell clustering and dimensionality reduction.

\subsection{Screening of differentially expressed genes}

We downloaded the standardized data-series matrix from GEO, and calculated the differentially expressed genes to be screened by the $\mathrm{R}$ language using the T-test (Student's t-test) statistical method. The screening conditions: $\mathrm{p}$-value $<0.05$; fold change $>1.5$ or $<0.667$.

\subsection{Functional enrichment analysis of differentially expressed genes}

Gene Ontology Database is referred to as GO database. It describes genes and protein functions in detail in a tree-like hierarchical manner to clarify the hierarchical relationship between gene functions. GO Analysis is a method based on the $\mathrm{GO}$ database to accurately targeted screen out the gene functions that represent the target gene group significantly. We used GO Analysis method to annotate the gene function of the differential genes, and got all the functions that the gene participates in. Subsequently, we used Fisher's exact test and multiple comparison test to calculate the significance level ( $p$-value) and false positive rate (FDR) of each function, so as toscreen out the notable function embodied by the differential genes. The criteria for significance screening: $p$-value $<0.05$.

\subsection{Signal pathway analysis of differentially expressed genes}

Kyoto Encyclopedia of Genes and Genomes (KEGG) is a database that systematically analyzes the relationship between genes, gene functions and genome information, which helps to study genes and expression information as a whole network for research. Pathway analysis was based on the KEGG database and was performed by using Fisher's exact test and chi-square test for differential genes to analyze the significance of the pathway in which the target gene participates, and screening was done according to $p$-value $<0.05$ to obtain significant pathways.

\subsection{Ligand-Receptor Pair Database}


The ligand-receptor pairs analyzed in this study came from public data sets provided by previous studies [23]. The ligand-receptor analysis was performed using the cellphonedb function provided by the cellphonedb database.

\subsection{Cell clustering, dimensionality reduction and visualization}

We used the "FindVariableGenes" function in the Seurat software package to extract the eigenvalues of highly variable genes (HVGs) for cell clustering and dimensionality reduction. Then we used the "RunPCA" function to perform principal component analysis on HVGs.

In order to remove the signal-to-noise ratio, we utilized the "ElbowPlot" function to select important principal components. Then, we used the "FindClusters" function to cluster the cells in the PCA space. We set the parameter resolution to 0.8 and only identified main cell types, such as T cells, B cells, or macrophages. We used the "RunUMAP" function to project the clustered cells into a two-dimensional space, and employed "DimPlot" function to realize the visualization of the clustering results.

\subsection{Identification of cell types}

In order to label cell clusters, we first executed the "FindMarker" function through the Seurat software package to identify the differentially expressed genes on each cluster. Then we used the transcriptome data set of the cell types to infer the cell type of each single cell through Single R, and annotate the cell population.

\subsection{Analysis of the interaction between cells}

We used the cellphonedb function provided by the cellphonedb database (www.CellPhoneDB.org) for cellcell interaction analysis [24]. The threshold for screening was $p$ value $<0.01$.

\section{Results}

\subsection{Differential gene expression in the decidua parietalis of PE}

So far, more and more studies have shown that abnormal decidualization is an important factor leading to PE [6, 25-29]. However, the abnormal interaction between decidual cells based on the ligand-receptor relationship in PE is still unclear. Therefore, we tested the differentially expressed genes (DEGs) in the decidua parietalis of the four groups of PE (GSM2480233-NS, GSM2480234-NS, GSM2480235-NS, GSM2480236-NS) and the normal control group (GSM2480225-NS, GSM2480226-NS, GSM2480227-NS, GSM2480228-NS). The results showed that compared with the control group, the gene expression in the decidua parietalis of PE was significantly different (Fig. 1A). In detail, 548 genes in the decidua parietalis of PE were significantly up-regulated and 533 genes were significantly down-regulated (Fig. 1B). In order to better study the genes significantly differentially expressed in $P E$, we further analyzed the functions of 
up-regulated and down-regulated DEGs and the signal pathways they are involved through GO and KEGG analysis.

GO analysis of the down-regulated DEGs showed that in terms of biological processes, the most enriched GO item is cellular response to chemokine. In addition, in terms of cell components, the most enriched GO item is sarcoplasmic reticulum. More importantly, protein complex involved in cell adhesion is also abundantly enriched. Moreover, the most abundant GO item in molecular functions is cytokine binding. Receptor ligand activity and chemokine activity are also significantly enriched (Fig. 1C). Through the KEGG signal pathway analysis of the down-regulated DEGs, 19 signal pathways were found to be significantly enriched. Among them, the most abundant signaling pathway is cytokine-cytokine receptor interaction. Besides, the TNF signaling pathway, IL-17 signaling pathway, TGF-beta signaling pathway, NF-kappa B signaling pathway and chemokine signaling pathway are also significantly enriched (Fig. 1C).

Through GO analysis of the up-regulated DEGs, it is obvious that in terms of molecular function, carbohydrate binding is most significantly enriched, while extracellular matrix structural constituent, growth factor binding and transmembrane receptor protein kinase activity are also significantly enriched in molecular function. In terms of cellular components, HOPS complex is the most enriched. Basement membrane is also significantly enriched in cell components, which is consistent with previous our research results. In addition, in terms of biological processes, the regulation of mRNA stability and the regulation of actins polymerization or depolymerization are significantly enriched (Fig. 1D). We also analyzed the up-regulated DEGs by KEGG signaling pathway and found that among 23 significantly enriched signaling pathways, focal adhesion is the most significant enrichment, and calcium signaling pathway, TGF-beta signaling pathway, phospholipase D signaling pathway and Ras signaling pathway are also significantly enriched.

Overall, these results indicate that the down-regulated DEGs are mainly concentrated in chemokines and immune-related pathways. The up-regulated DEGs may play an important function in the extracellular matrix. As we all know, there are abundant regulatory factors with immunomodulatory activity in the extracellular matrix, such as growth factors [30] and chemokines [31]. These factors are closely related to the cell-cell interaction of immune cells. Therefore, the results of the study indicate that these DEGs play a vital role in the immune regulation process in the decidua.

\subsection{Abnormal function of decidual macrophages in PE}

Known from the above results, in the decidua parietalisof PE patients, DEGs affect the cell-cell interaction of immune cells. Therefore, we further studied the cell-to-cell interaction which was specifically affected by the abnormal expression of the differential genes. Cell populations form a potential cell-cell interaction network among specific protein complexes, which are based on the relationship between receptor and ligand genes (Fig. 2A). Therefore, we compared the genes contained in down-regulated DEGs and upregulated DEGs and in the ligand-receptor pairs that interact with immune cells, and found that 2 down- 
regulated DEGs (ICAM1 and CXCL3) and 6 up-regulated DEGs (IGF1, CXCL12, NRP1, LRP6, PDGFD and PDGFRB) are expressed in a ligand-receptor relationship (Fig. 2B \& Fig. 2C).

Through the analysis of single-cell sequencing data, we found that ICAM1 and CXCL3 are both expressed in normal dM, but IGF1, CXCL12, NRP1, LRP6, PDGFD and PDGFRB are not only not expressed in normal $\mathrm{dM}$, but also not expressed in other immune cells (Fig. 3). Then, based on the ligand-receptor relationship that these DEGs participate in, we explored the cell-to-cell interaction (Table 2). We found one characteristics: most of them exist in the interrelationship between $\mathrm{dM}$ and other decidual immune cells (Fig. 4), which indicates that abnormal gene expression affects the interaction of $\mathrm{dM}$ with other decidual immune cells.

\subsection{The cell-to-cell interaction of decidual macrophages}

To explore the function of $\mathrm{dM}$, we further investigated the interaction of $\mathrm{dM}$ with other decidual immune cells using single-cell sequencing. The single cell data in decidua comes from the GEO database, which contains three normal samples, corresponding to 9066 cells. In order to standardize the data, we performed data quality control and deleted some cells with expression genes less than 200 and greater than 6000 (UMI count greater than 0 and greater than 6000) and the percentage of mitochondrial genes greater than $5 \%$ (Fig. 5A). As the amount of sequencing data increases, the number of genes detected in a single cell increases and the number of UMls also increases, indicating that there is a positive correlation between genes and UMI (Fig. 5B). However, there is no correlation between the number of genes and the percentage of mitochondria, which means that the number of genes is not affected by the percentage of mitochondria (Fig. 5C). After the correction of the expression matrix, the number of non-variable genes was 12264, while the number of mutant genes was 3000 , therefore, the number of mutant genes was far less than the number of non-variable genes (Fig. 5D). We selected 2000 HVGs from the corrected expression matrix and extracted their feature values for cell clustering and dimensionality reduction. After the principal component analysis of 2000 HVGs, 19 principal components were finally determined through permutation test. As we all know, each type of cell contains a different number and type of genes. Therefore, according to this characteristic, the principal components of the cells were analyzed and projected into the two-dimensional space to achieve the purpose of cell dimensionality reduction. The results showed that cells with the same characteristics will eventually be gathered together, and on the contrary, cells without the same characteristics will be far apart (Fig. 5E).

In order to further investigate the type of cells, we identified the DEGs in each cell population (Fig. 6A), and selected known cell marker genes from them. Under the influence of biological background, by consulting the literature, we can explore the related genes of each cell type and their expression in different cell types. We found that several cell markers are specifically expressed in only one cell, such as FGFBP2 and MYOM2 are only expressed in dNK-e cells; TPSB2 and TPSAB1 are only expressed in Mast cells; IGLC2 and CD79A are only expressed in B cells. In addition, other cell markers are expressed in a variety of cells, but their expression levels are higher in one cell (Fig. 6B). 
Therefore, we used known cell marker genes expressed in different cell types to define each cell cluster. We combined cell populations expressing the same cell markers and observed that dNK cells are the most abundant in decidua, and dM cells are second only to dNK cells and CD8 T cells. In addition, there are fewer B cells, mast cells, and ILC3 cells (Fig. 6C). In order to systematically study the interaction between various immune cells in the decidua, we used CellPhoneDB to analyze the interaction between them. The results showed that $\mathrm{dM}$ plays a role through the export function and interacts most closely with other immune cells. Abnormal dM can affect the functions of other cells, such as ILC3 cells, dNK-a cells, dNK-b cells, dNK-d cells, and CD4T cells, while the functions of dNK-a cells and dNK-b cells are affected by a variety of cells (Fig. 6D). In summary, the results indicate that the interaction between the $\mathrm{dM}$ and other immune cells plays an important role in the establishment and maintenance of the entire pregnancy process. Therefore, the abnormal exportation of $\mathrm{dM}$ affects the role of immune cells interplaying with them, consequently jeopardizing the original immune regulation, and ultimately causing the occurrence of PE.

\section{Discussion}

In this study, we used conventional expression profiling to compare the DEGs contained in decidua parietalis of the PE and normal human. We further explored their involvement in cell-to-cell interactions using CellPhoneDB analysis. We found that most of differential genes exist in the interrelationship between decidual macrophages and other decidual immune cells. We further used single-cell sequencing and discovered that $\mathrm{dM}$ affect the functions of other immune cells in the decidua through exporting function. So, the abnormal exportation of $\mathrm{dM}$ due to abnormal expression of differential genes affects the role of immune cells interplaying with them, consequently impeding the original immune regulation, and ultimately causing the occurrence of PE.

Most previous studies on the pathogenesis of PE have been focused on placenta, including placental ischemia and placental dysfunction. However, decidualization is the foundation for placentation and growth. Abnormal decidualization of the endometrium can lead to embryo implantation failure or abnormal implantation, which in turn leads to the occurrence of $P E[8,9]$. In this process, there are a series of immune regulatory mechanisms. Thereinto, both tumor necrosis factor (TNF) and nuclear factor-kB (NF-kB) are situated at the central link in the network of immune regulation [32,33]. The TNF is a central polypeptide mediator of the cellular immune response, it participates in TNF signaling pathway, and can recognize and participate in the activation of NF-KB $[34,35]$. In PE patients, the balance of NF-KB signaling pathway is disrupted, leading to abnormal apoptosis of placental trophoblast cells [36, 37]. In this research, we found that CXCL3 and ICAM1 participate in the TNF signaling pathway and NF-KB signaling pathway. In addition, these two genes are highly expressed in normal $\mathrm{dM}$, but are significantly down-regulated in PE. At the same time, we also found that genes that are significantly up-regulated in the decidua parietalis of $\mathrm{PE}$ are not only not expressed in $\mathrm{dM}$, but also not expressed in other normal decidual immune cells, including IGF1, CXCL12, NRP1, LRP6, PDGFD, and PDGFRB. These results indicate that the abnormal export function of $\mathrm{dM}$ affects the functions of immune cells that interact with them, 
thereby destroying the original immune regulation mechanism, and ultimately leading to the occurrence of PE.

Previous studies have demonstrated that CXCL3 and CXCL12 are chemokines, they can coordinate the migration, positioning and the interaction between immune cells $[31,38]$. In addition, CXCL3 can inhibit the proliferation of T cells and increase the apoptosis of T cells, while IGF1 has the opposite effect [39, 40]. In detail, IGF1 can inhibit T cell apoptosis, and promote T cell autophagy [40]. CXCL12 activates PKA, $\mathrm{CREB}, \mathrm{Bcl} 2$ and $\mathrm{BcIXI}$, thereby prolonging the survival time of $\mathrm{CD} 4 \mathrm{~T}$ lymphocytes. In addition, it promotes the accumulation of NK cells in the uterus by enhancing the adhesion of NK cells to decidual stromal cells $[39,41]$. ICAM1 is a single-chain transmembrane glycoprotein that can promote the proliferation of naive CD $4 \mathrm{~T}$ cells and also plays a key role in the interaction between antigen presenting cells and $\mathrm{T}$ cells [42]. NRP1 can regulate the transfer of CD4T cells and allow the acceptance of allogeneic grafts, making NRP1 a new target for immunotherapy [43]. It is worth noting that the Wnt signaling pathway which LRP6 is involved in regulates the differentiation and development of various immune cells such as macrophages, $T$ cells and $B$ cells, and regulates the immune response process through various mechanisms [44-47]. Overall, the above-mentioned DEGs play an important role in the immune regulation. The abnormal expression of these DEGs can cause the disorders of the immune system and participate in the occurrence of PE.

Interestingly, we found that these DEGs have one thing in common: according to the ligand-receptor relationship network, these DEGs are all present in the export founction of $\mathrm{dM}$. As the second largest type of leukocyte at the maternal-fetal interface, $\mathrm{dM}$ exerts anti-inflammatory and phagocytosis effects in the early pregnancy, and by regulating the local immune microenvironment of the decidua, remodels the decidual blood vessels, participates in embryo implantation and maintains pregnancy and other processes $[22,48]$. In the decidua of PE patients, the increased expression of TGF- $\beta 3$ activates miR-494 in decidual mesenchymal stem cells, thereby weakening the regulation of mesenchymal stem cells, thereby turning macrophages into M2 type, and finally leads to the immune imbalance of maternal-fetal interface [49]. Imbalanced ratio of M1/M2 macrophages is considered to be one of the causes of pregnancyrelated diseases, including $P E$, fetal growth restriction (FGR) and preterm delivery $[18,50]$. Our research found that dM affects the function of ILC3 cells, dNK-a cells, dNK-b cells, dNK-d cells, and CD4T cells through export in the decidua. Therefore, the abnormal export of $\mathrm{dM}$ affects the function of immune cells that interact with it, thereby destroying the original immune regulation mechanism.

Although $\mathrm{dM}$ is not the focus of most studies on the pathogenesis of $\mathrm{PE}$, our research has found that DEGs that appear in the export of dM play a key role in the development of PE. To be precise, the activation of NRP1 can affect angiogenesis, and it activates the intracellular kinase ABL1 independently of the VEGF signaling pathway [51]. Whereas abnormal angiogenesis is an important factor leading to PE [52]. LRP6 regulates the autophagy mediated by Rab7, and then regulates the invasion and migration of trophoblasts through the Wnt/ $\beta$-catenin pathway $[44,45]$. Insufficient invasion of extravillous trophoblast can lead to impaired uterine spiral artery remodeling and subsequent PE [53]. IGF1 is one of the genes 
related to the insulin signaling pathway, and its up-regulation may cause polycystic ovary syndrome and endometrial cancer [54]. However, in PE, the impact of IGF up-regulation is unclear.

In summary, our research has found that the imbalance of immune regulation caused by abnormal $\mathrm{dM}$ function plays an important role in the pathogenesis of $P E$. These findings provide new insights for studying the pathogenesis of $\mathrm{PE}$, and also give new ideas for the diagnosis and treatment of PE.

However, how do CXCL3, ICAM1, IGF1, CXCL12, NRP1 and LRP6 as ligands or receptors affect the export function of $\mathrm{dM}$, which in turn affects the function of other decidual immune cells, thereby destroying the original immune regulation mechanism, and ultimately leading to the occurrence of $P E$, which needs to be further investigated.

\section{Conclusion}

Our results show that abnormal gene expression affects the export function of decidual macrophages, which in turn affects the function of immune cells interacting with them, thereby destroying the original immune regulation mechanism, and ultimately leading to the occurrence of preeclampsia.

\section{Declarations}

\section{Acknowledgements}

This study was supported by grants from the National KeyR\&D Program of China (2017YFC1001403, 2019YFA0802600), and NSFC (31871512and 31671199) to CZ. Support was also obtained by the Shanghai Commission of Science and Technology (17DZ2271100).

\section{Authors' contributions}

Study design: ZC. Data analysis: ZC, YXY, ZHY and RMM. Data interpretation: RMM, YXY, ZHY, ZC and ZC. Drafting of the manuscript: RMM. Revision of the manuscript: ZC. All authors contributed significantly to this work and agreed to be accountable for the work. All authors read and approved the final manuscript.

\section{Funding}

There is no funding associated with this manuscript.

\section{Availability of data and materials}

The datasets supporting the conclusions of this article are available in the GEO repository (https://www.ncbi.nlm.nih.gov/geo). GEO accession GSE130560 (https://www.ncbi.nlm.nih.gov/geo/query/acc.cgi?acc=GSE130560) and GSE94643 (https://www.ncbi.nlm.nih.gov/geo/query/acc.cgi?acc=GSE94643).

\section{Ethics approval and consent to participate}


Not applicable.

\section{Consent for publication}

Not applicable.

\section{Competing interests}

The authors declare no competing interests.

\section{Authors' information}

${ }^{1}$ Shandong Provincial Key Laboratory of Animal Resistance Biology, College of Life Sciences, Shandong Normal University, Ji'nan, Shandong, China; ${ }^{2}$ School of Medicine, Xiamen University, Xiamen, 361102, Fujian, China; ${ }^{3}$ Center for Reproductive Medicine, Ren Ji Hospital, School of Medicine, Shanghai Jiao Tong University, Shanghai, China; ${ }^{4}$ Shanghai Key Laboratory for Assisted Reproduction and Reproductive Genetics, Shanghai, China.

\section{References}

1. Report of the National High Blood Pressure Education Program Working Group on High Blood Pressure in Pregnancy. AM J OBSTET GYNECOL. 2000; 183:s1-s22.

2. Correa PJ, Palmeiro Y, Soto MJ, Ugarte C, Illanes SE. Etiopathogenesis, prediction, and prevention of preeclampsia. Hypertens Pregnancy. 2016; 35:280-294.

3. Staff AC. The two-stage placental model of preeclampsia: An update. J Reprod Immunol. 2019; 134135:1-10.

4. Steegers EAP, von Dadelszen P, Duvekot JJ, Pijnenborg R. Pre-eclampsia. The Lancet. 2010; 376:631644.

5. Ellis R, Katerelos M, Choy SW, Cook N, Lee M, Paizis K, Pell G, Walker S, Power DA, Mount PF. Increased expression and phosphorylation of 6-phosphofructo-2-kinase/fructose-2,6-bisphosphatase isoforms in urinary exosomes in pre-eclampsia. J Transl Med. 2019; 17.

6. Gellersen B, Brosens JJ. Cyclic decidualization of the human endometrium in reproductive health and failure. Endocr Rev. 2014; 35:851-905.

7. Liu H, Huang X, Mor G, Liao A. Epigenetic modifications working in the decidualization and endometrial receptivity. Cell Mol Life Sci. 2019; 77:2091-2101.

8. Ramathal CY, Bagchi IC, Taylor RN, Bagchi MK. Endometrial decidualization: of mice and men. Semin Reprod Med. 2010; 28:17-26.

9. Maruyama TaYY. Molecular and Cellular Mechanisms for Differentiation and Regeneration of the Uterine Endometrium. Endocr J. 2008; 55: 795-810. 
10. Coulam C. What about superfertility, decidualization, and natural selection? J Asst Rep Gen. 2016; 33:577-580.

11. Liu S, Diao L, Huang C, Li Y, Zeng Y, Kwak-Kim JYH. The role of decidual immune cells on human pregnancy. J Reprod Immunol. 2017; 124:44-53.

12. Yang F, Zheng Q, Jin L. Dynamic Function and Composition Changes of Immune Cells During Normal and Pathological Pregnancy at the Maternal-Fetal Interface. Front Immunol. 2019; 10.

13. Olmos-Ortiz A, Flores-Espinosa P, Mancilla-Herrera I, Vega-Sánchez R, Díaz L, Zaga-Clavellina V. Innate Immune Cells and Toll-like Receptor-Dependent Responses at the Maternal-Fetal Interface. Int J Mol Sci. 2019; 20.

14. Bonney EA. Immune Regulation in Pregnancy. Obstet Gynecol Clin North Am. 2016; 43:679-698.

15. Solano ME. Decidual immune cells: Guardians of human pregnancies. BEST PRACT RES CL OB. 2019; 60:3-16.

16. Fu B, Wei H. Decidual natural killer cells and the immune microenvironment at the maternal-fetal interface. Sci China: Life Sci. 2016; 59:1224-1231.

17. Li Y, Lopez GE, Vazquez J, Sun Y, Chavarria M, Lindner PN, Fredrickson S, Karst N, Stanic AK. Decidual-Placental Immune Landscape During Syngeneic Murine Pregnancy. Front Immunol. 2018; 9.

18. Zhang J, Dunk C, Croy AB, Lye SJ. To serve and to protect: the role of decidual innate immune cells on human pregnancy. Cell Tissue Res. 2015; 363:249-265.

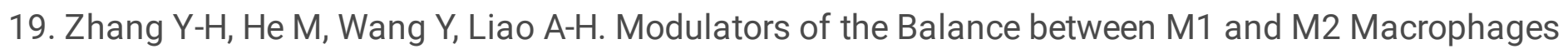
during Pregnancy. Front Immunol. 2017; 8.

20. Murray Peter J, Allen Judith E, Biswas Subhra K, Fisher Edward A, Gilroy Derek W, Goerdt S, Gordon S, Hamilton John A, Ivashkiv Lionel B, Lawrence T, et al. Macrophage Activation and Polarization: Nomenclature and Experimental Guidelines. Immunity. 2014; 41:14-20.

21. Li Z-H, Wang L-L, Liu H, Muyayalo KP, Huang X-B, Mor G, Liao A-H. Galectin-9 Alleviates LPS-Induced Preeclampsia-Like Impairment in Rats via Switching Decidual Macrophage Polarization to M2 Subtype. Front Immunol. 2019; 9.

22. Houser BL. Decidual Macrophages and Their Roles at the Maternal-Fetal Interface. J Biol Med. 2012; 85:105-118.

23. Ramilowski JA, Goldberg T, Harshbarger J, Kloppmann E, Lizio M, Satagopam VP, Itoh M, Kawaji H, Carninci P, Rost B, Forrest AR. A draft network of ligand-receptor-mediated multicellular signalling in human. Nat Commun. 2015; 6:7866.

24. Vento-Tormo R, Efremova M, Botting RA, Turco MY, Vento-Tormo M, Meyer KB, Park JE, Stephenson E, Polanski K, Goncalves A, et al. Single-cell reconstruction of the early maternal-fetal interface in humans. Nature. 2018; 563:347-353.

25. Garrido-Gomez T, Dominguez F, Quinonero A, Diaz-Gimeno P, Kapidzic M, Gormley M, Ona K, PadillaIserte $\mathrm{P}, \mathrm{McMaster} \mathrm{M}, \mathrm{Genbacev} \mathrm{O}$, et al. Defective decidualization during and after severe 
preeclampsia reveals a possible maternal contribution to the etiology. Proc Natl Acad Sci U S A. 2017; 114:E8468-E8477.

26. Conrad KP, Rabaglino MB, Post Uiterweer ED. Emerging role for dysregulated decidualization in the genesis of preeclampsia. Placenta. 2017; 60:119-129.

27. Garrido-Gomez T, Fisher SJ, Simón C. Reply to Liu et al.: Decidualization defect in severe preeclampsia. Proc Natl Acad Sci. 2018; 115:E7656-E7657.

28. Lv H, Tong J, Yang J, Lv S, Li W-P, Zhang C, Chen Z-J. Dysregulated Pseudogene HK2P1 May Contribute to Preeclampsia as a Competing Endogenous RNA for Hexokinase 2 by Impairing Decidualization. Hypertension. 2018; 71:648-658.

29. Rabaglino MB, Conrad KP. Evidence for shared molecular pathways of dysregulated decidualization in preeclampsia and endometrial disorders revealed by microarray data integration. The FASEB Journal. 2019; 33:11682-11695.

30. Travis MA, Sheppard D. TGF- $\beta$ Activation and Function in Immunity. Annu Rev Immunol. 2014; 32:5182.

31. Luster CLSaAD. The chemokine system in innate immunity. Cold Spring Harb Perspect Biol. $2015 ; 7$.

32. Holbrook J, Lara-Reyna S, Jarosz-Griffiths H, McDermott MF. Tumour necrosis factor signalling in health and disease. F1000Research. 2019; 8.

33. Sun S-C. The non-canonical NF-kB pathway in immunity and inflammation. Nat Rev Immunol. 2017; 17:545-558.

34. Van Quickelberghe E, De Sutter D, van Loo G, Eyckerman S, Gevaert K. A protein-protein interaction map of the TNF-induced NF-KB signal transduction pathway. Scientific Data. 2018; 5.

35. STEFAN SCHUTZE':' KW, THOMAS MACHLEIDT \and MARTIN KRONKE':'. TNF-Induced Activation of NF-xB. Journal. 1995; 193: 193-203.

36. MERAL ABAN LC, KAPLANOGLU, RABIA ARPACI, MURAT ARSLAN UD, MUSTAFA and SAFFET DILEK. Expression of Nuclear Factor-Kappa B and Placental Apoptosis in Pregnancies Complicated with Intrauterine Growth Restriction and Preeclampsia: An Immunohistochemical Study. Journal. 2004:204 (203), 195-202.

37. Ignacio Caballero SAG, Shaghayegh Basatvat, Javier A. Sa'nchez-Lo' pez, Mehrnaz Montazeri, Nasim Maslehat, Sarah Elliott, Neil R. Chapman, Alireza Fazeli*. Human Trophoblast Cells Modulate Endometrial Cells Nuclear Factor KB Response to Flagellin In Vitro. PLoS ONE. 2013; 8.

38. Dubinett SM, Lee JM, Sharma S, Mulé JJ. Chemokines. The Cancer Journal. 2010; 16:325-335.

39. Lee Y-S, Won K-J, Park S-W, Lee H-W, Kim B, Kim J-H, Kim D-K. Mesenchymal stem cells regulate the proliferation of T cells via the growth-related oncogene/CXC chemokine receptor, CXCR2. Cell Immunol. 2012; 279:1-11.

40. Wang F, Tan Y-Q, Zhang J, Zhou G. Insulin-like growth factor 1 exhibits the pro-autophagic and antiapoptotic activity on T cells of oral lichen planus. Int J Biol Macromol. 2019; 133:640-646. 
41. Patrick Bellelis ea. Transcriptional changes in the expression of chemokines related to natural killer and T-regulatory cells in patients with deep infiltrative endometriosis. ENDOMETRIOSIS. 2013;99:1987-1993.

42. He Wei ML, Chao Wen, Anying Zhang, Kun Yang, Hong Zhou, Xinyan Wang. Identification of an intercellular cell adhesion molecule-1 homologue from grass carp: Evidence for its involvement in the immune cell adhesion in teleost. Fish Shellfish Immunol. 2018; 81:67-72.

43. Mauricio Campos-Mora RAM, Francisco Pérez, Tania Gajardo, Javier Campos, Diego Catalan, Juan Carlos Aguillón and Karina Pino-Lagos. Neuropilin-1+ regulatory T cells promote skin allograft survival and modulate effector CD4+ T cells phenotypic signature. Immunology and Cell Biolog. 2014:1-7.

44. Li L, Peng W, Zhou Q, Wan JP, Wang XT, Qi HB. LRP6 regulates Rab7-mediated autophagy through the Wnt/ $\beta$-catenin pathway to modulate trophoblast cell migration and invasion. J Cell Biochem. 2019.

45. Yu X, Zhang Y, Yang P, Gao X, Wang Y. Downregulated low-density lipoprotein receptor-related protein 6 induces the maldevelopment of extravillous trophoblast via Wnt/ $\beta$-catenin signaling pathway. Mol Cell Probes. 2019; 44:21-28.

46. Chae W-J, Bothwell ALM. Canonical and Non-Canonical Wnt Signaling in Immune Cells. Trends Immunol. 2018; 39:830-847.

47. Haseeb, Pirzada, Ain, Choi. Wnt Signaling in the Regulation of Immune Cell and Cancer Therapeutics. Cells. 2019; 8.

48. Ning F, Liu H, Lash GE. The Role of Decidual Macrophages During Normal and Pathological Pregnancy. Am J Reprod Immunol. 2016; 75:298-309.

49. Zhao G, Miao H, Li X, Chen S, Hu Y, Wang Z, Hou Y. TGF-ß3-induced miR-494 inhibits macrophage polarization via suppressing PGE2 secretion in mesenchymal stem cells. FEBS Lett. 2016; 590:16021613.

50. Schust. The Contribution of Macrophages to Normal and Pathological Pregnancies. Am J Reprod Immunol. 2010; 63: 460-471.

51. Lampropoulou A, Ruhrberg C. Neuropilin regulation of angiogenesis. Biochem Soc Trans. 2014; 42:1623-1628.

52. Liu H, Li Y, Zhang J, Rao M, Liang H, Liu G. The defect of both angiogenesis and lymphangiogenesis is involved in preeclampsia. Placenta. 2015; 36:279-286.

53. Winship AL, Koga K, Menkhorst E, Van Sinderen M, Rainczuk K, Nagai M, Cuman C, Yap J, Zhang J-G, Simmons $D$, et al. Interleukin-11 alters placentation and causes preeclampsia features in mice. Proc Natl Acad Sci. 2015; 112:15928-15933.

54. Shafiee MN, Seedhouse C, Mongan N, Chapman C, Deen S, Abu J, Atiomo W. Up-regulation of genes involved in the insulin signalling pathway (IGF1, PTEN and IGFBP1) in the endometrium may link polycystic ovarian syndrome and endometrial cancer. Mol Cell Endocrinol. 2016; 424:94-101. 


\section{Tables}

Table 1. Summary of abbreviations.

\begin{tabular}{|ll|}
\hline Abbreviations & Full name \\
\hline PE & Preeclampsia \\
\hline IGFBP1 & insulin-like growth factor binding protein 1 \\
PRL & prolactin \\
dNK & decidual natural killer cells \\
dM & decidual macrophages \\
DC & dendritic cells \\
GEO & Gene Expression Omnibus \\
FDR & false positive rate \\
KEGG & Kyoto Encyclopedia of Genes and Genomes \\
HVGs & highly variable genes \\
DEGs & differentially expressed genes \\
TNF & tumor necrosis factor \\
NF-KB & nuclear factor-KB \\
FGR & fetal growth restriction \\
\hline
\end{tabular}

Table 2. The cell-to-cell interactions of differentially expressed genes involved. 
inter interacting_pair inter ligand receptor

\begin{tabular}{|lllll|}
\hline CXCL12_CXCR3 & dM|dNK_d & 0.388 & CXCL12 & CXCR3 \\
CXCL12_CXCR3 & dNK_eldNK_d & 0.37 & CXCL12 & CXCR3 \\
CXCL12_CXCR4 & dM|dNK_d & 1.365 & CXCL12 & CXCR4 \\
CXCL12_CXCR4 & dM|CD4 T & 1.298 & CXCL12 & CXCR4 \\
CXCL3_CXCR2 & dM|ILC3 & 0.49 & CXCL3 & CXCR2 \\
CXCL3_CXCR2 & dM|dNK_e & 0.49 & CXCL3 & CXCR2 \\
CXCL3_CXCR2 & dM|dNK_e & 0.49 & CXCL3 & CXCR2 \\
CXCL3_CXCR2 & dM|Mast cell & 0.49 & CXCL3 & CXCR2 \\
DPP4_CXCL12 & CD4 T|dNK_e & 0.103 & DPP4 & CXCL12 \\
DPP4_CXCL12 & CD4 T|dNK_a & 0.097 & DPP4 & CXCL12 \\
ICAM1_AREG & dM|dNK_d & 1.533 & ICAM1 & AREG \\
ICAM1_AREG & dM|dNK_b & 1.475 & ICAM1 & AREG \\
IGF1_IGF1R & dM|dNK_e & 0.135 & IGF1 & IGF1R \\
IGF1_IGF1R & dM|dNK_d & 0.134 & IGF1 & IGF1R \\
LRP6_CKLF & dM|dNK_a & 0.975 & LRP6 & CKLF \\
LRP6_CKLF & dM|dNK_c & 0.962 & LRP6 & CKLF \\
NRP1_PGF & dM|ILC3 & 0.194 & NRP1 & PGF \\
NRP1_PGF & dM|dNK_b & 0.187 & NRP1 & PGF \\
NRP1_VEGFA & dM|Mast cell & 0.47 & NRP1 & VEGFA \\
NRP1_VEGFA & Mast cell|dM & 0.45 & NRP1 & VEGFA \\
NRP1_VEGFB & dM|dNK_a & 0.209 & NRP1 & VEGFB \\
NRP1_VEGFB & dM|ILC3 & 0.207 & NRP1 & VEGFB \\
PDGFRB_PDGFD & dNK_eldNK_d & 0.077 & PDGFRB & PDGFD \\
PDGFRB_PDGFD & Mast cell|dNK_d & 0.056 & PDGFRB & PDGFD \\
\hline
\end{tabular}

$\mathrm{dM}$, decidual macrophages; dNK, decidual natural killer cells; ILC, innate lymphoid cells. DC, dendritic cells. 
Figures

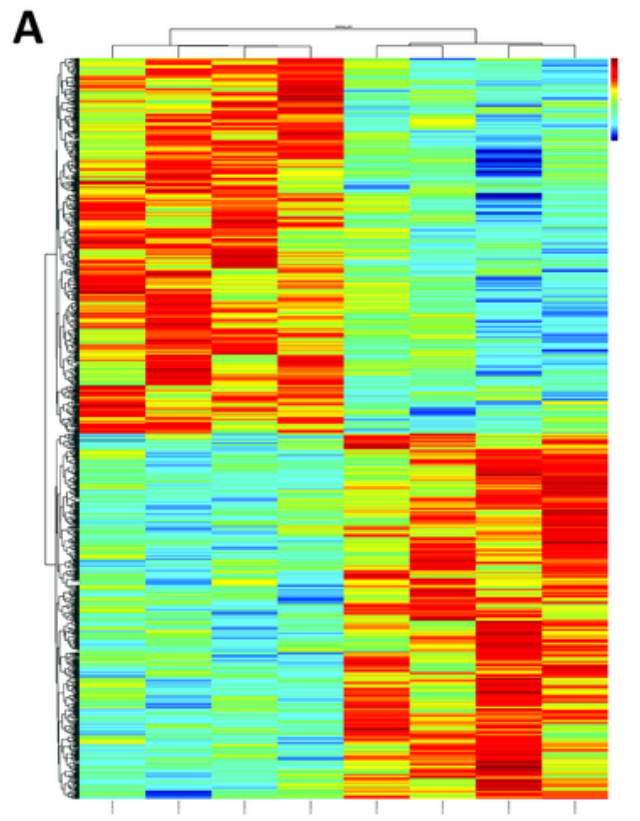

B

- Up-regulated genes

- Down-regulated genes

- Not differential expressed genes

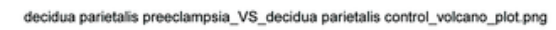

C
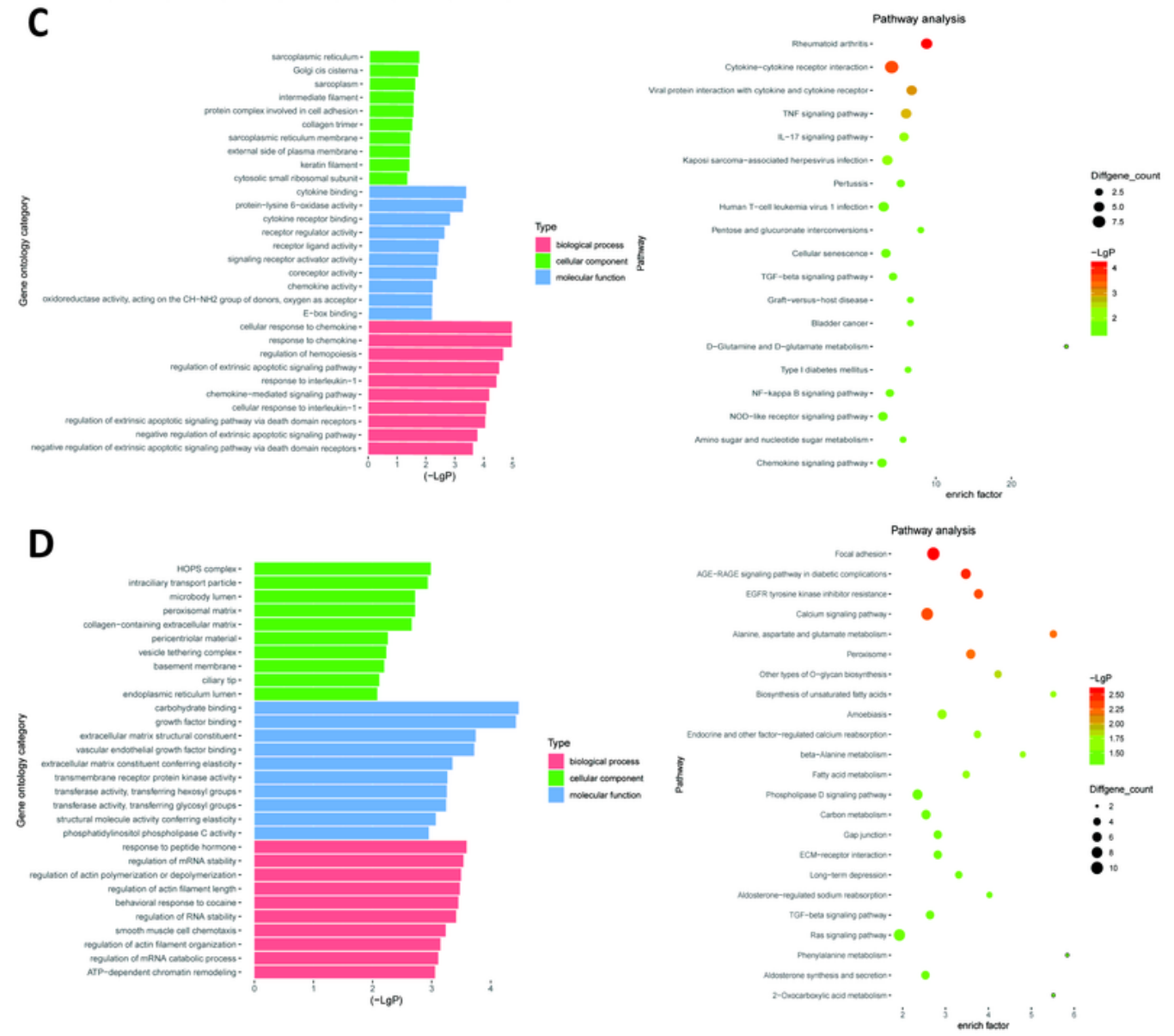

Figure 1

Differential gene expression in the decidua parietalis between PE and control group. A. The heatmap represents the differentially expressed genes (DEGs) in four groups of the decidua parietalis of PE (GSM2480233-NS, GSM2480234-NS, GSM2480235-NS, and GSM2480236-NS) and normal control group 
(GSM2480225-NS, GSM2480226-NS, GSM2480227-NS, and GSM2480228-NS) with p-value $<0.05$, fold change $>1.5$ or $<0.667$. Each row represents a DEG; Differential expression levels were illustrated by the pseudo color. Red color indicates the genes in PE were up-regulated than the control group; green $\llbracket$ downregulated. B.Volcano figure showing the significantly DEGs in the decidua parietalis of the PE in comparison to controls.A red dot represents up-regulated DEGs with log2 ratio $\geq 1.5$ and $p$-value $<0.05$; a green dot represents down-regulated DEGs with log2 ratio $\leq-1.5$ and $p$-value $<0.05$. C. GO analysis and KEGG pathway enrichment for the down-regulated DEGs. Left panel: GO functional classification of the down-regulated DEGs. Green, blue, and red represent the three classes of the GO term. The top ten enriched GO terms were shown in each class. Right panel: Scatter plot for the KEGG enrichment of the down-regulated DEGs. The enrich factor is the ratio of the down-regulated DEGs in a pathway to all the genes in this pathway.The top 19 pathways were shown with p-value< 0.05 in the figure. D. GO analysis and KEGG pathway enrichment for the up-regulated DEGs. Left panel: GO functional classification of the up-regulated DEGs. Green, blue, and red represent the three classes of the GO term. The top ten enriched GO terms were shown in each class. Right panel: Scatter plot for the KEGG enrichment of the up-regulated DEGs. The top 23 pathways were shown with $p$-value $<0.05$.
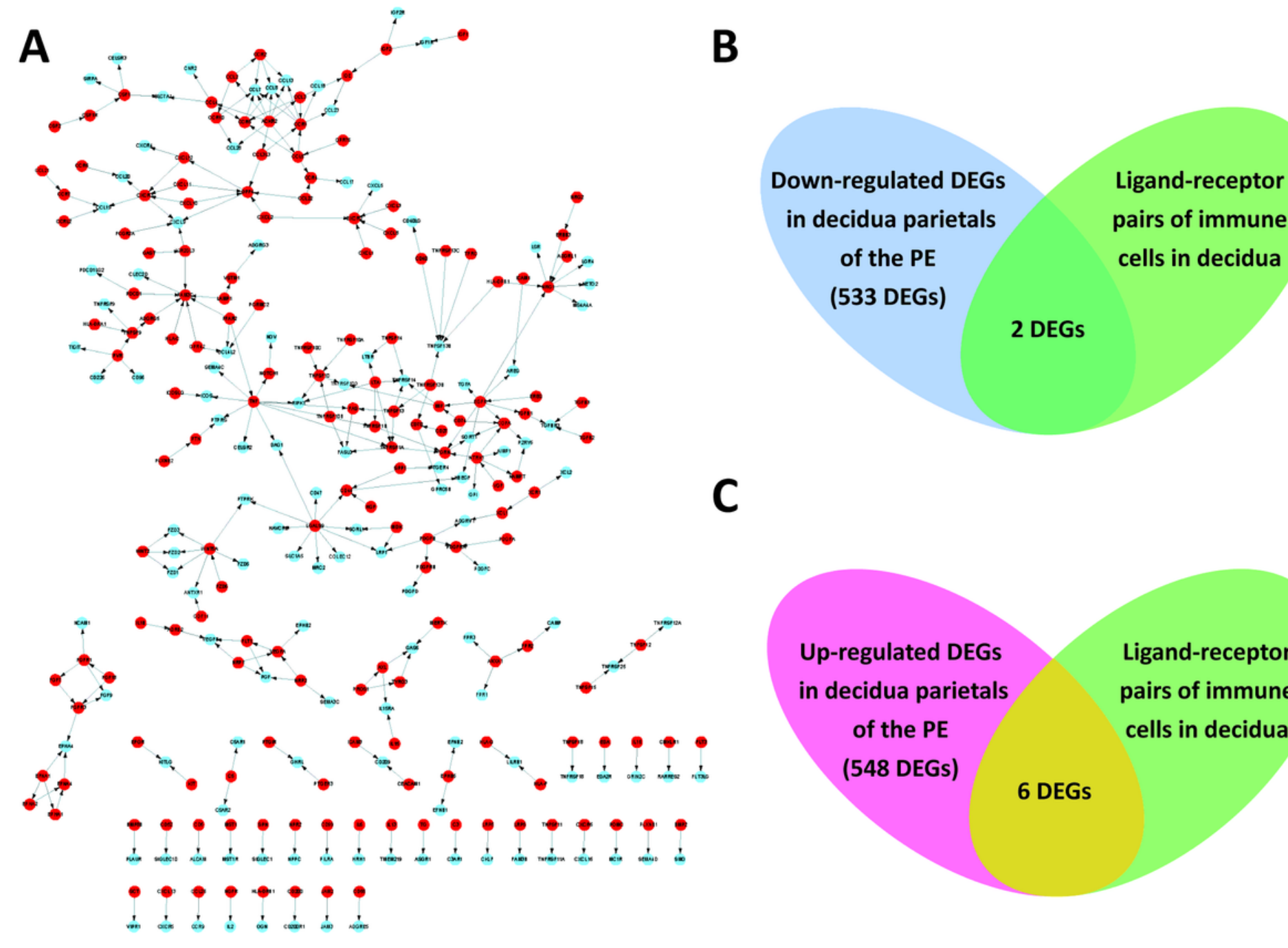
(533 DEGs)

2 DEGs

Up-regulated DEGs

in decidua parietals

of the PE

(548 DEGs)
Ligand-receptor pairs of immune cells in decidua

\section{DEGs}

Figure 2 
The ligand-receptor relationshipsof thethe differentially expressed genes (DEGs) in the decidua parietalis of PE. A. The ligand-receptor signaling network of immune cells in deciduas.Red dots represent ligands and blue dots represent receptors. Thearrows points from ligands to receptors. B.Venn diagrams of the significantly down-regulated DEGs in the decidua parietalis of the PE and in the ligand-receptor pairs of immune cells in deciduas.C.Venn diagrams of the significantly up-regulated DEGs in the decidua parietalis of the PE and in the ligand-receptor pairs of immune cells in decidua.

A
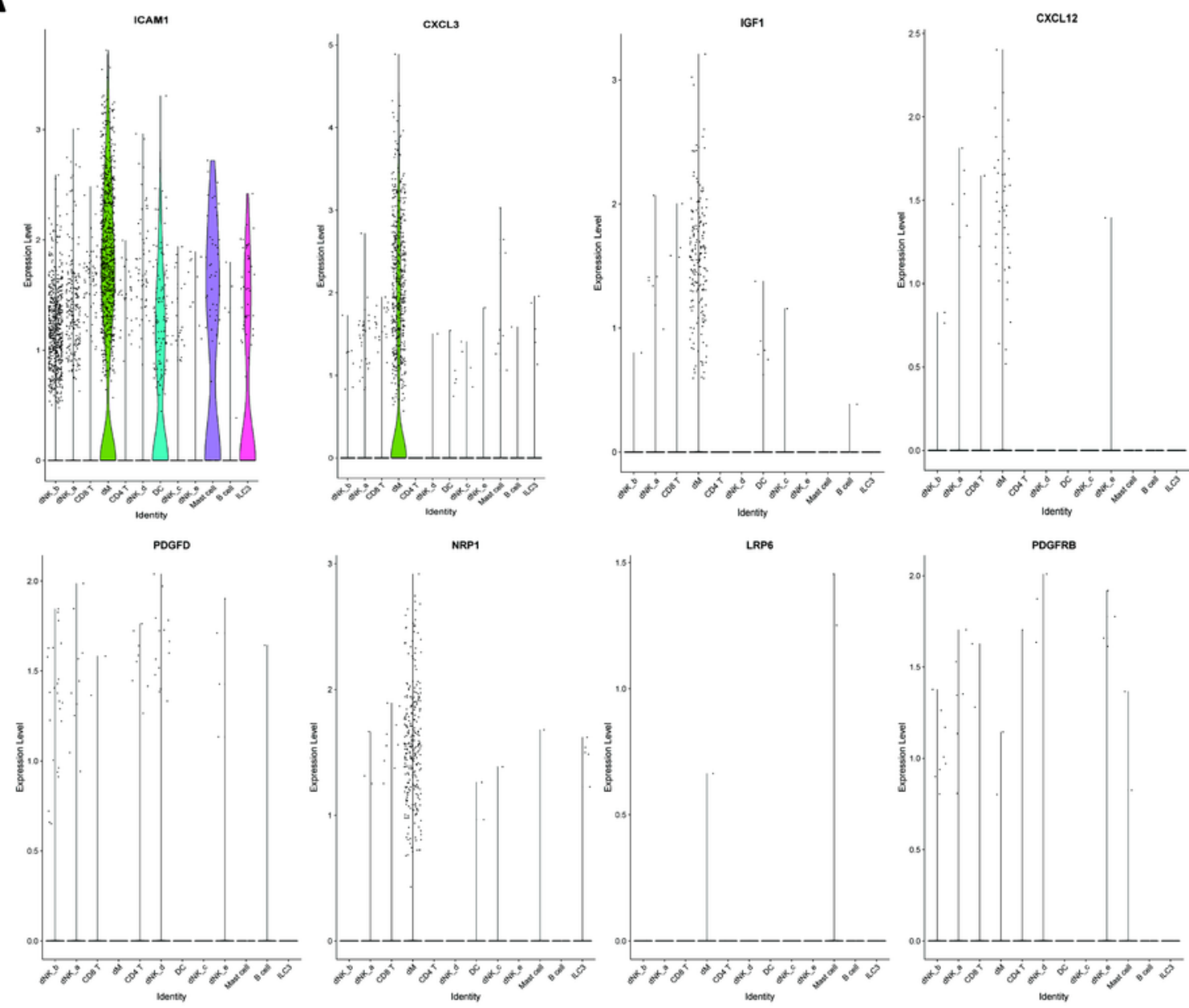

B
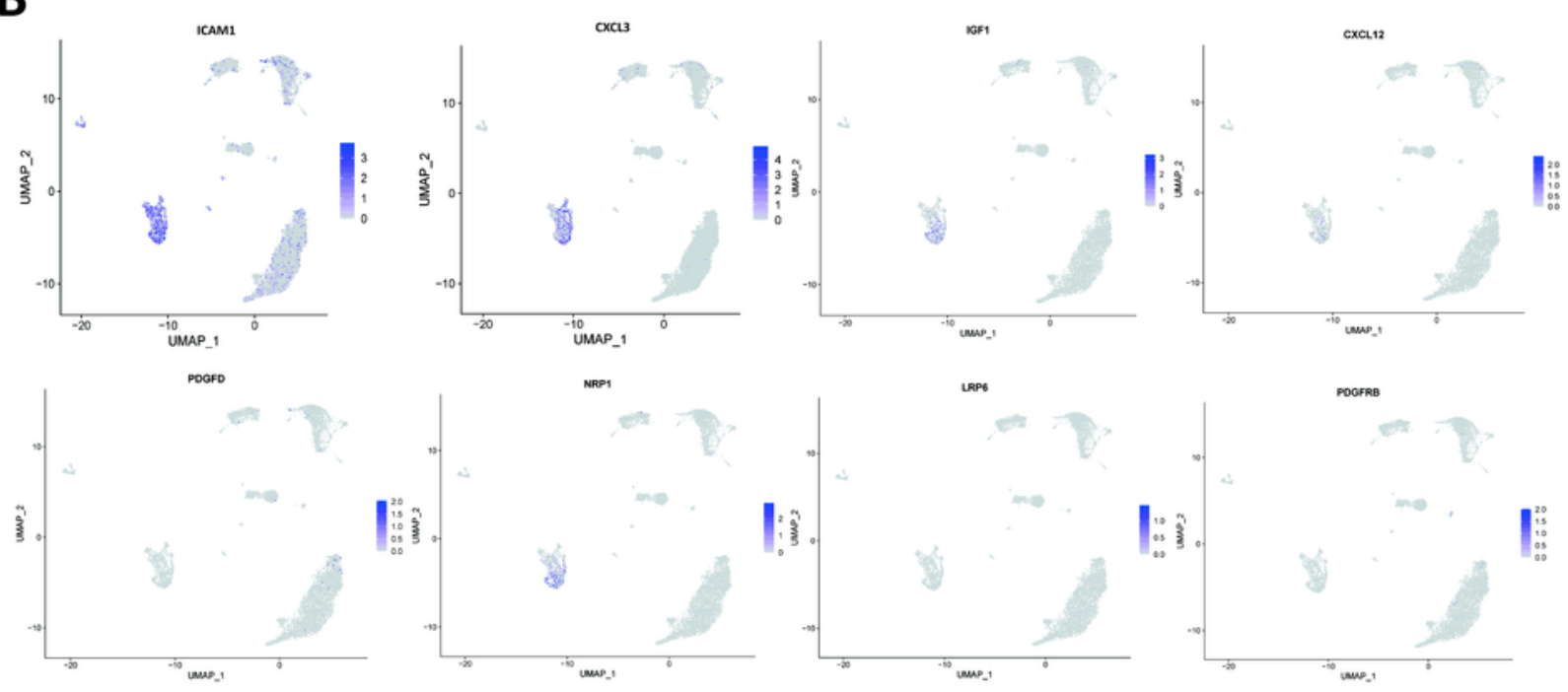

Figure 3 
The expression of DEGs in the immune cells of normal decidua. A. The violin plots showing the expression of two down-regulated DEGs (ICAM1 and CXCL3) and six up-regulated DEGs (IGF1, CXCL12, NRP1, LRP6, PDGFD, and PDGFRB) in the immune cells of normal decidua.Cellsfrom Fig. 2c are used for the violin plots. B. The UMAP visualization maps of various immune cell clustersin the normal deciduas by single cell RNA sequencing analysis. These maps show the expression of down- regulated and upregulated DEGs in various immune cells. Cell clusters from Fig. $2 \mathrm{c}$ are used for the UMAP visualization maps. dM, decidual macrophages; dNK, decidual natural killer cells; ILC, innate lymphoid cells. DC, dendritic cells.

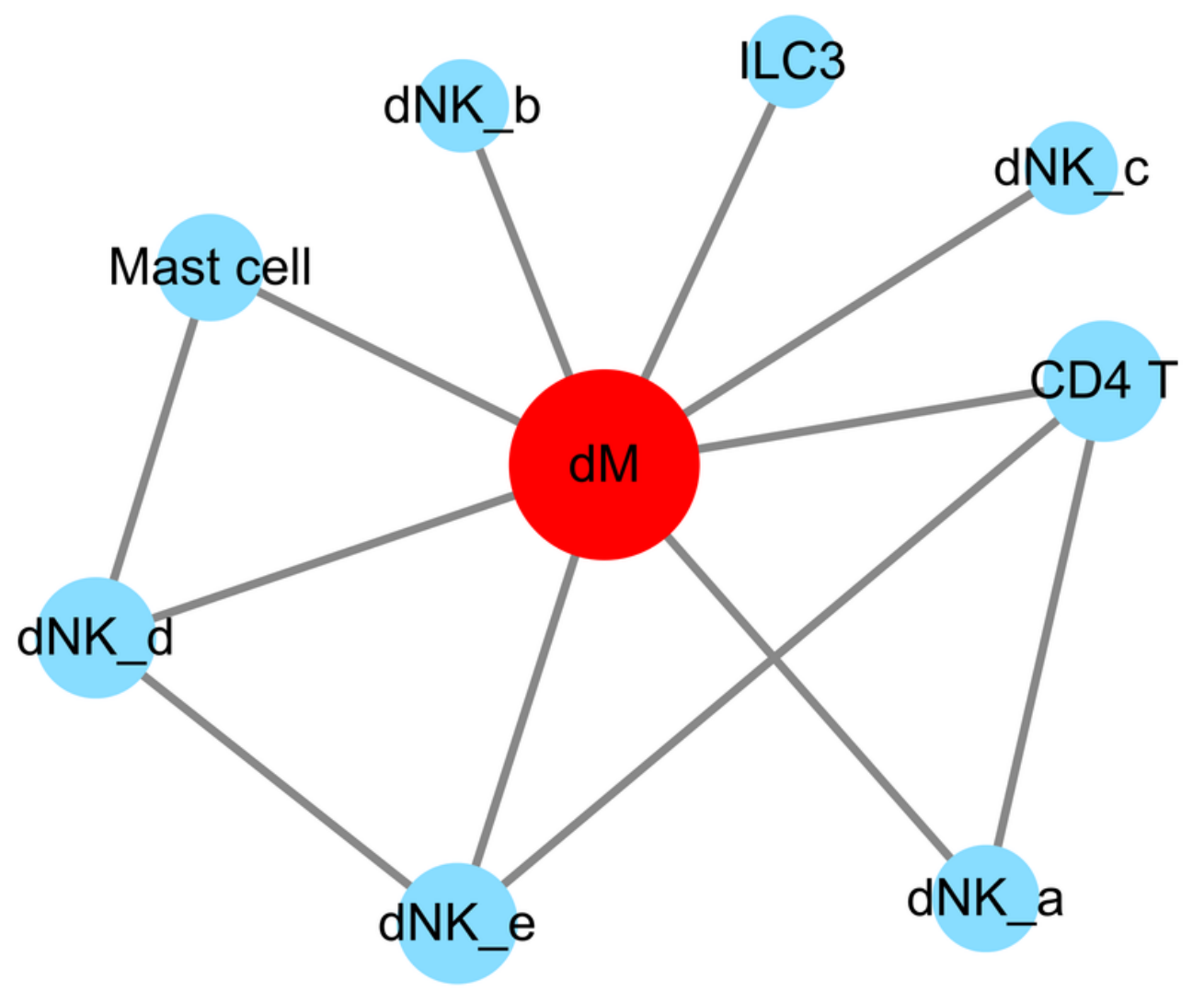

Figure 4

The cell-to-cell interactionsof DEGs involved.The nodes are cell types and the segments are cell-to-cell interactions. The red node indicates cell types that are highly interacted with other cells. The size of the different cell types is proportional to the amount of total number of interactions with the red node. $\mathrm{dM}$,decidual macrophages; dNK,decidual natural killer cells; ILC,innate lymphoid cells. 

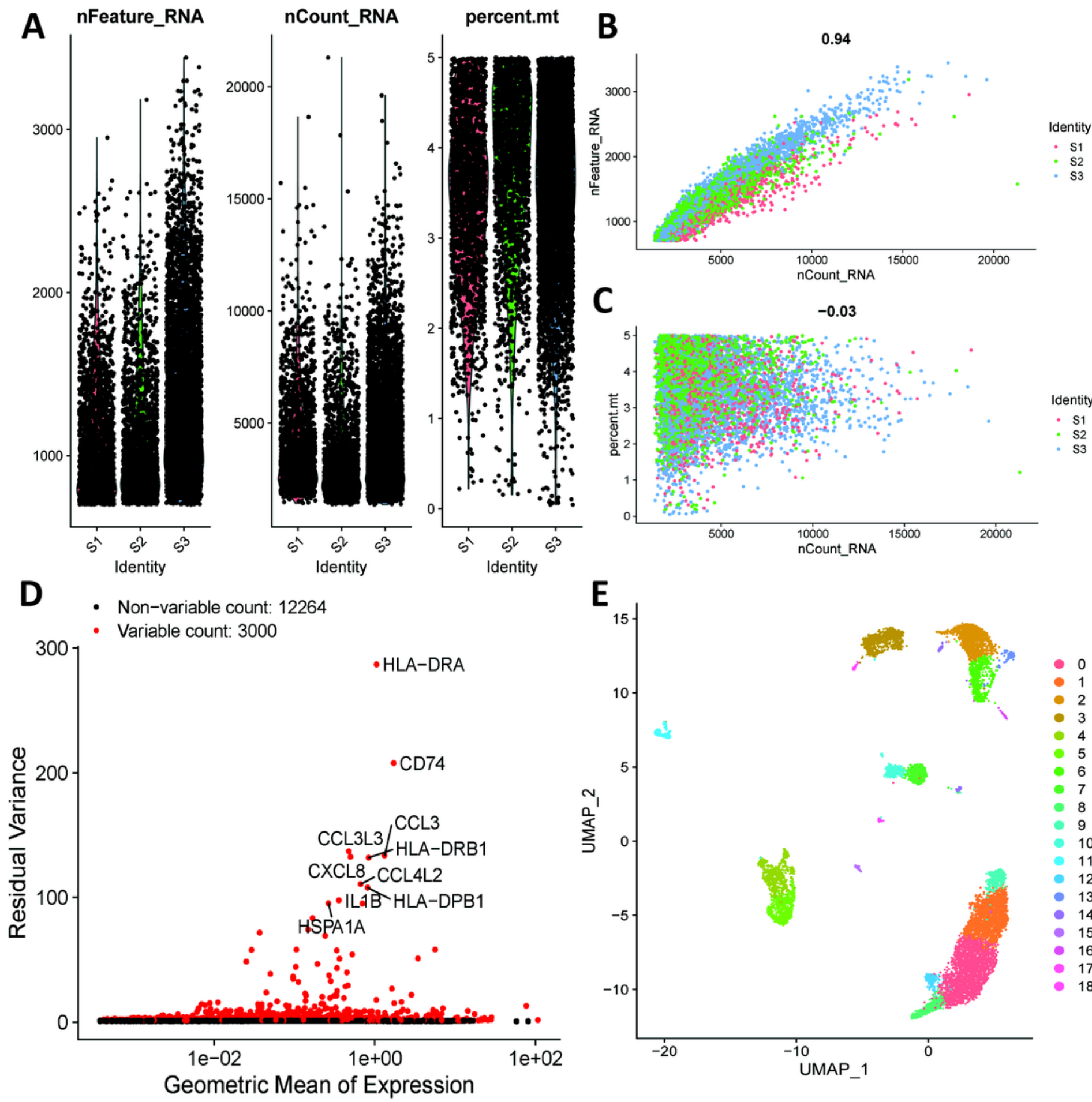

$\mathbf{E}$

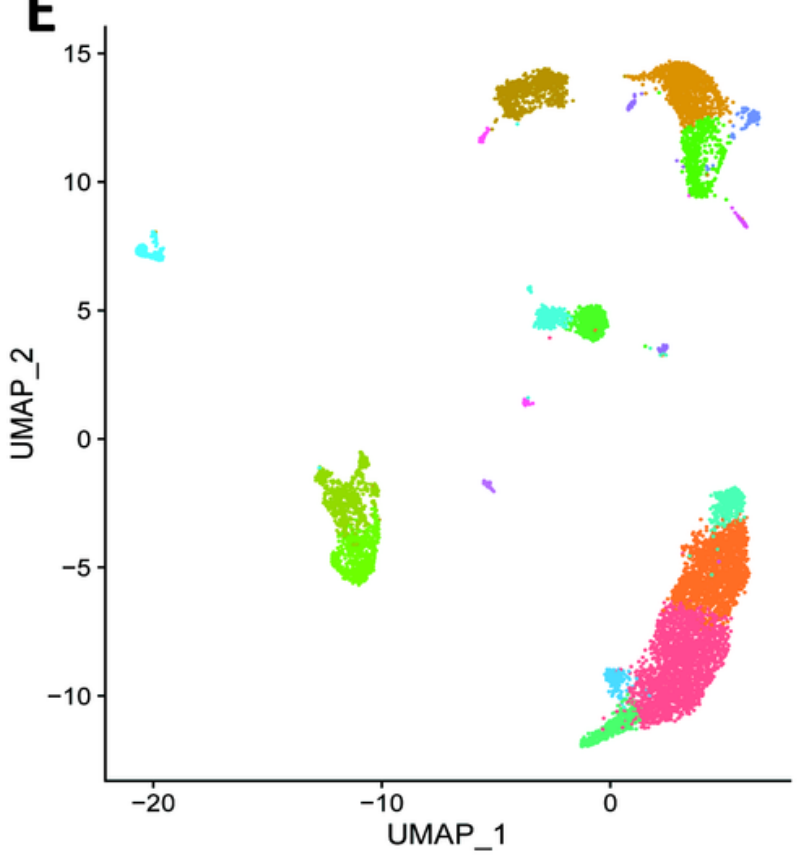

Figure 5

Overview of preparations for cell type identification in deciduas. A. Gene expression in each of the 9066 cells in three normal samples (S1, S2, and S3). The left graph shows the number of genes contained in each cell.The middle figure indicates the confidence level of the data.The right graph shows the percentage of mitochondria.The black dots represent cells. B. The relevance of the number of UMI and the number of genes.The horizontal coordinate is the number of UMI of the cell and the vertical coordinate is the number of genes in the corresponding cell.The red, green and blue dots respectively 
represent samples S1, S2 and S3.C. The relevance of the number of UMI and the percentage of mitochondria. The horizontal coordinate is the number of UMI of the cell and the vertical coordinate is the percentage of mitochondria.The red, green and blue dots respectively represent samples S1, S2 and S3.D. Theanalytical map of genetic variability. Red dots represent variable counts and black dots represent nonvariable counts. E.The UMAP visualization maps of 19 cell clusters in the deciduas by single cell RNA sequencing analysis.

A

B

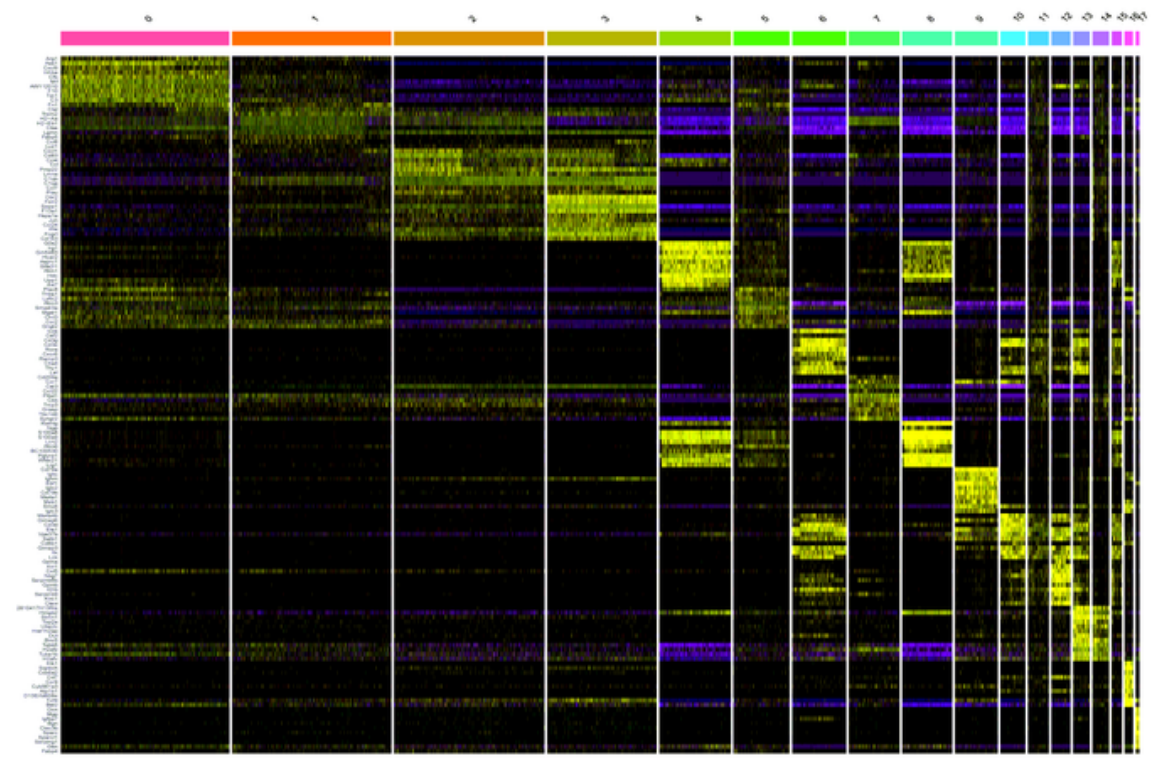

B
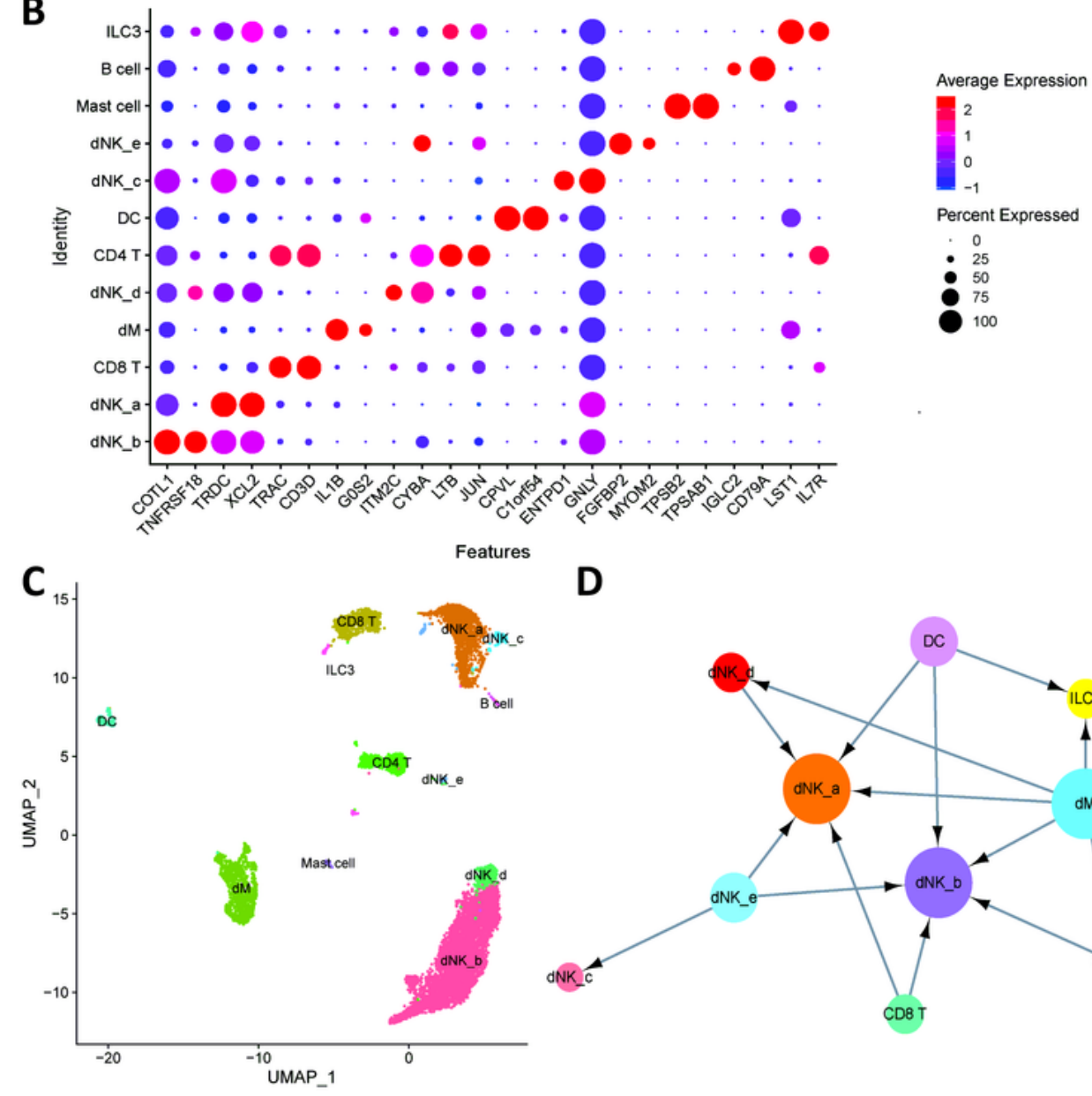

D

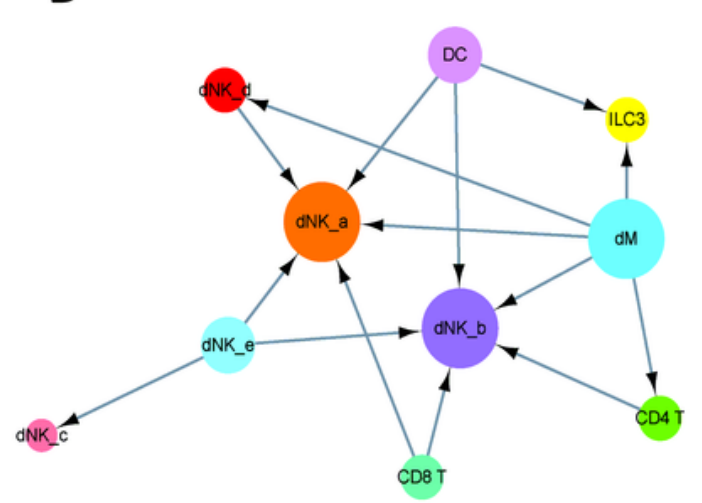

Figure 6 
The identification and interactions of decidual cell clusters by single-cell sequencing. A. The heatmap showing the expression of differential genesin separate cell clusters of normal deciduas. B. The dot plot reveals the expression of some known markers in each immune cell clusters of normal deciduas. The size of the ball represents percent expressed. The color of the ball represents average expression. C. The UMAP visualization maps of various immune cell clusters in the normal deciduas by single cell RNA sequencing analysis. The various colors represent different types of cells. D. The cell-to-cell interaction analysis was conducted by CellPhoneDB.Cell types are presented by nodes and interactions are indicated by arrows. The size of the nodes represents the number of cells contained in each cell type in the deciduas ( $p$-value < 0.01).->, output role; <-, input role. dM, decidual macrophages; dNK, decidual natural killer cells; ILC, innate lymphoid cells. DC, dendritic cells. 